\title{
All-Optical Basic Arithmetic Elements using Dark-Bright Solitons Conversion Control
}

\author{
Somsak Mitatha ${ }^{\mathrm{a},{ }^{*},}$ Khanthanou Luangxaysana ${ }^{\mathrm{a}}$, Preecha Yupapin ${ }^{\mathrm{b}}$ \\ ${ }^{a}$ Hybrid Computing Research Laboratory, Faculty of Engineering, \\ King Mongkut's Institute of Technology Ladkrabang, Bangkok, 10520 THAILAND \\ ${ }^{b}$ Nanoscale Science and Research Alliance (N'SEAR), Faculty of Science, \\ King Mongkut's Institute of Technology Ladkrabang, Bangkok 10520, THAILAND \\ *Corresponding Author: kmsomsak@hotmail.com
}

\begin{abstract}
In this paper, we propose a new design for all-optical basic arithmetic elements using optical technique namely dark-bright solitons conversion control within an optical add-drop filter. In operation, all-optical data input logic ' 0 ', ' 1 ' is formed by dark soliton (D) and bright soliton (B) pulses, respectively. The conversion behaviour between dark and bright soliton pulses can be obtained and formed the logic pulses by a $\pi / 2$ phase shifted device such as an optical coupler, in which the binary logic operation can be formed simultaneously at the thought and drop ports, respectively. In all-optical NAND gate and S-R flip-flop application can be recognized as a simple and flexible system for forming the logic switching system. Numerical simulation results are obtained and confirmed for the useful application, in which the advanced logical system can be operated.
\end{abstract}

Keywords: all-optical, Digital optics, Add-drop filter.

\section{Introduction}

Near future, computation and communication problems are inevitable since conventional electronic technology will be soon reaching its speed limit. All optical logic gates are the important key element in next generation optical computing and in networking to perform optical signal processing such as binary addition, header reorganization, parity checking, optical bit pattern recognition addressing, demultiplexing, regenerating and switching with very high speed. Thus, the use of light signals has been proposed for signal processing instead of electrons because the electric current flows at only 10 percent of the speed of light. By using light, the optical components for digital processing are required to fulfill the full applications. Till date, the practical components and optical signal processing are still some years away. The optical techniques have been widely increasing and applying for many optical applications for communication use. One of those applications is a processing system. An all-optical flip-flop is the packet switches in the next generation of communication network. The flip-flop is a logic gates that maintain a stable state output even after the input is turned off, in which the key basic function is represented by the logic gates. Nowadays, All-optical flip-flop is an essential component for latching functions in ultrahigh speed all-optical processing applications $^{(1,2)}$. Currently, many systems such as a terahertz optical asymmetric de-multiplex (TOAD) ${ }^{(3)}$, semiconductor optical amplifier $^{(4)}$, Fabry-Perot laser diode ${ }^{(5)}$, ring lasers ${ }^{(6)}$, photonic crystals ${ }^{(7,8)}$, DBR laser $\operatorname{diode}^{(9)}$ have been proposed. In this paper, we have presented an all-optical flip-flop system for logical processing operation and arithmetic operation base tree architecture, which can be used for an electronic circuit replacement, which can be replaced used for an electronic circuit ${ }^{(10-13)}$ by using dark-bright soliton pulses conversion control ${ }^{(14-15)}$, the theoretical background is also reviewed. For our concept, the all-optical flip-flop operations and arithmetic operations of binary based on dark-bright solitons conversion behaviors can be performed, in which the coincidence dark and bright soliton pulses can be separated after propagating into the optical-filter device with $\pi / 2$ phase shifted (an optical coupler) ${ }^{(14)}$. The proposed scheme is based on a 1 bit binary to the complex logic circuits, which can be 
compared by any 2 bits, when logic ' 0 ' and ' 1 ' are formed by dark and bright soliton pulses, respectively.

In this paper, we propose an all-optical circuit for logic NAND Gate, S-R flip-flop and arithmetic operations. The theoretical background is also reviewed. In this concept, the simultaneous logic operation of binary based on dark-bright soliton conversion behaviors can be performed, in which the coincidence dark and bright soliton pulses can be separated after propagating into the $\pi / 2$ phase shifted device (an optical coupler). The proposed scheme is based on a 1 bit binary compared to the complex logic circuits, which can be compared by any 2 bits, when logic ' 0 ' and ' 1 ' use the dark and bright soliton pulses, respectively.

\section{Dark-Bright Solitons Conversion Control}

The operation of dark-bright solitons conversion ${ }^{(16)}$ using a ring resonator as shown in Figure 1, where in this case the dark-bright solitons conversion system using an optical channel dropping filter (OCDF) is composed of two set of coupled waveguide, as shown in Figure 1 (a) and (b), when for convenience, Figure 1 (b) is replaced by Figure 1 (a). The relative phase of the two output light signals after coupling into the optical coupler is $\pi / 2$ before coupling into the ring and the input bus, respectively. This means that the signals coupled into the drop and through ports are acquired a phase of $\pi$ with respect to the input port signal. In application, if the coupling coefficients are formed appropriately, the field coupled into the through port would completely extinguish the resonant wavelength, and all power would be coupled into the drop port, in which the dark-bright conversion behaviors are described by Eqs. (1) to $(8)$.

$$
\begin{aligned}
& E_{r a}=-j K_{l} E_{i}+\tau_{l} E_{r d} \\
& E_{r b}=\exp (j \omega T / 2) \exp (-\alpha L / 4) E_{r a} \\
& E_{r c}=\tau_{2} E_{r b}+j K_{2} E_{r a} \\
& E_{r d}=\exp (j \omega T / 2) \exp (-\alpha L / 4) E_{r c} \\
& E_{t}=\tau_{1} E_{i}-j K_{l} E_{r d} \\
& E_{d}=\tau_{2} E_{a}-j K_{2} E_{r b}
\end{aligned}
$$

where $E_{i}$ is the input field, $E_{a}$ is the added (control) field, $E_{t}$ is the throughput field, $E_{d}$ is the dropped field, $E_{r a} \ldots E_{r d}$ are the fields in the ring at the point $a \ldots d, K_{l}$ is the field coupling coefficient between the input and the ring, $K_{2}$ is the field coupling coefficient between the ring and the output bus, $L$ is the circumference of the ring $(2 \pi R), T$ is the time taken for one round trip, and $\alpha$ is the power loss in the ring per unit length. We assume that lossless coupling, i.e. $\tau_{1,2}=\sqrt{1-\kappa_{1,2}^{2}}, T$ is the round-trip time $T=L n_{\text {eff }} / c$. The output power-intensities at the drop port and through port are given by

$$
\begin{aligned}
& \left|E_{d}\right|^{2}=\left|\frac{-\kappa_{1} \kappa_{2} A_{1 / 2} \Phi_{1 / 2}}{1-\tau_{1} \tau_{2} A \Phi} E_{i}+\frac{\tau_{2}-\tau_{1} A \Phi}{1-\tau_{1} \tau_{2} A \Phi} E_{a}\right|^{2} \\
& \left|E_{t}\right|^{2}=\left|\frac{\tau_{2}-\tau_{1} A \Phi}{1-\tau_{1} \tau_{2} A \Phi} E_{i}+\frac{-\kappa_{1} \kappa_{2} A_{1 / 2} \Phi_{1 / 2}}{1-\tau_{1} \tau_{2} A \Phi} E_{a}\right|^{2}
\end{aligned}
$$

where $A_{1 / 2}=\exp (-\alpha L / 4)$ (the half-round-trip amplitude).

$A=A_{1 / 2}^{2}, \Phi_{1 / 2}=\exp (j \omega T / 2) \ldots$ (the half-round-trip phase contribution), and $\Phi=\Phi_{1}^{2}$. The input and control fields at the input and add ports are formed by the dark-bright optical solitons as shown in Eqs. (9) - (10).

$$
\begin{gathered}
E_{\text {in }}(t)=A_{0} \operatorname{sech}\left[\frac{T}{T_{0}}\right] \exp \left[\left(\frac{z}{2 L_{D}}\right)-i \omega_{0} t\right] \\
E_{\text {in }}(t)=A_{0} \tanh \left[\frac{T}{T_{0}}\right] \exp \left[\left(\frac{z}{2 L_{D}}\right)-i \omega_{0} t\right]
\end{gathered}
$$

where $A$ and $z$ are optical field amplitude and propagation distance, respectively. $T$ is soliton pulse propagation time in a frame moving at the group velocity $T=t-\beta_{1} z$, where $\beta_{1}$ and $\beta_{2}$ are the coefficients of the linear and second-order terms of Taylor expansion of the propagation constant. $L_{D}=T_{0}^{2} /\left|\beta_{2}\right|$ is the dispersion length of the soliton pulse. $T_{0}$ in the equation is the initial soliton pulse width, where $t$ is the soliton phase shift time, and the frequency shift of the soliton is $\omega_{0}$. This solution describes a pulse that keeps its temporal width invariance as it propagates, and thus is called a temporal soliton. When a soliton peak intensity is given, then $T_{0}$ is known. For the soliton pulse in the nanoring device, a balance should be achieved between the dispersion length $\left(L_{D}\right)$ and nonlinear length $\left.L_{N L}=1 / \Gamma \phi_{N L}\right)$, where $\Gamma=n 2 k 0$, is the length scale over which dispersive or nonlinear effects make the beam become wider or narrower. For a soliton pulse, there is a balance between dispersion and nonlinear lengths, hence $L_{D}=L_{N L}$.

When light propagates within the nonlinear material (medium), the refractive index $(n)$ of light within the medium is given by Eq. (11)

$$
n=n_{0}+n_{2} I=n_{0}+\frac{n_{2}}{A_{e f f}} P
$$


here $n_{0}$ and $n_{2}$ are the linear and nonlinear refractive indexes, respectively. $I$ and $P$ are the optical intensity and optical power, respectively. The effective mode core area of the device is given by $A_{\text {eff. }}$. For the micro/nano ring resonator, the effective mode core areas range from 0.10 to $0.50 \mu \mathrm{m}^{2(6)}$. The resonant output of the light field is the ratio between the output and input fields $\left[E_{t}\right.$ and $\left.E_{i}\right]$ in each round trip, which is given by references ${ }^{(16)}$.

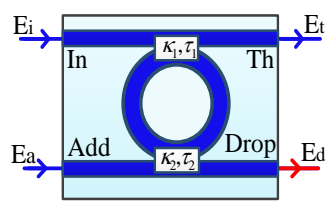

(a)

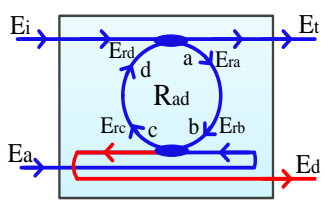

(b)
Figure 1. A schematic diagram of the dark-bright soliton conversion control system

\section{All Optical Logic NAND Gate}

The proposed all-optical logic NAND gates devise is as shown in Figure. 2. The input and control light pulse trains are input in to the first add/drop filter (No. "01") using the the dark-bright solitons, where firstly, the dark soliton is converted to be dark and bright solitons via add/drop filter, which can be seen at the through and drop ports with $\pi$ phase shift, respectively. By using add/drop filter (No. "11", "12"), both input signals are generated by the first stage add/drop filter. Next, the input data " $A$ " with logic " 0 " (dark soliton) and logic "1" (bright soliton) are added into both add ports. Secondly, the dark-bright soliton conversion with $\pi$ phase shift is operated again. Finally, by using add/drop filter (No. "21" and " 22 "), the input data "B" with logic " 0 " (dark soliton) and logic " 1 " (bright soliton) are seen at all the add ports. For large scale of circuit shown in Figure. 2, results obtained are simultaneously seen by D21, D22, T21, T22 at the drop and through ports for optical logic gates, respectively. The truth table of logic NAND gate is as shown in Figure. 3

In simulation, add/drop filter fixed parameters used are $R_{a d}=1.50 \mu \mathrm{m}, A_{e f f}=0.25 \mu \mathrm{m}^{2}, \alpha=0.05 \mathrm{~dB} / \mathrm{mm}$, the couple intensity loss is $\gamma=0.1, n_{0}=3.34(\operatorname{InGaAsP} / \operatorname{InP})$, the coupling coefficient of the micro-ring resonator is $k_{s}=0.5$.

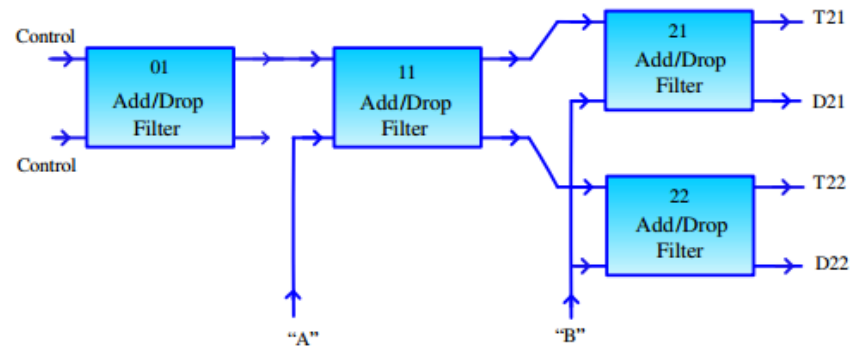

Figure 2. Schematic diagram simultaneous NAND gates

\begin{tabular}{|c|c|c|}
\hline Input A & Input B & Output \\
\hline 0 & 0 & 1 \\
\hline 0 & 1 & 1 \\
\hline 1 & 0 & 1 \\
\hline 1 & 1 & 0 \\
\hline
\end{tabular}

Figure 3. Truth table of logic NAND gate

The nonlinear refractive index is $n_{2}=2.2 \times 10^{-17}$ with center wavelength of $1.5 \mu \mathrm{m}$ and pulse width $20 \mathrm{~ns}$, power at $1 \mathrm{~W}$ is input into add/drop system.

Results of the NAND gate are generated by the dark-bright soliton conversion behaviors, the input data logic " 0 " and " 1 " are represented by the dark and bright soliton pulses, respectively. However, in practice, the time domain results can be obtained by using the term $T=1 / f$, where $T$ and $f$ are a period and frequency of the signal. In Figure. 4, Figure. 5, Figure. 6 and Figure. 7.

Case 1: When the input data logic " 00 " is added, the obtained output optical logic is "1000" (Figure. 4).

Case 2: When the input data logic " 01 " is added, the output optical logic "1010" (Figure. 5) is obtained.

Case 3: When the input data logic " 10 " is added, the output optical logic "1100" (Figure. 6) is obtained.

Case 4: When the input data logic " 11 " is added, we found that the output optical logic "0110" (Figure. 7) is seen.

From the simulation result, we found that output data logic at the through port T22 is optical NAND gate, the output data logic at drop port D21 and through port T22 are same with the input "A" and "B", respectively. 


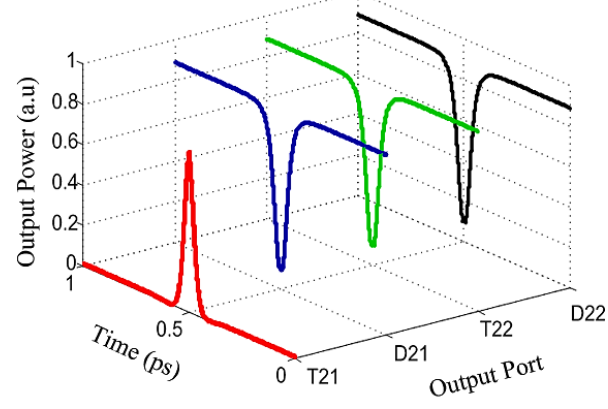

Figure 4. Show the output logic gate when the input logic stages ' 00 ' or 'DD'.

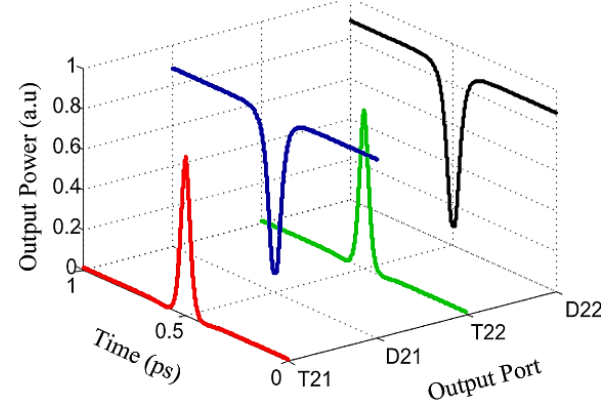

Figure 5. Show the output logic gate when the input logic stages ' 01 ' or 'DB'.

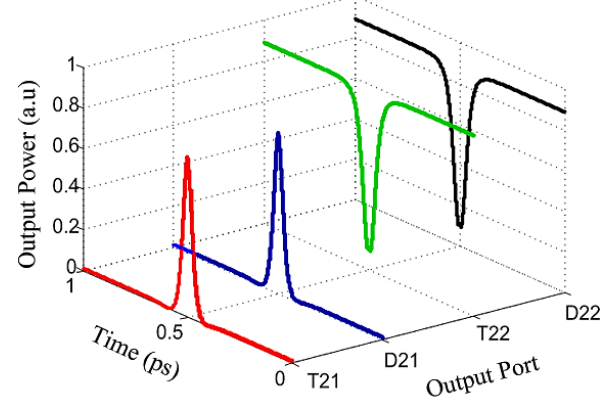

Figure 6. Show the output logic gate when the input logic stages ' 10 ' or 'BD'.

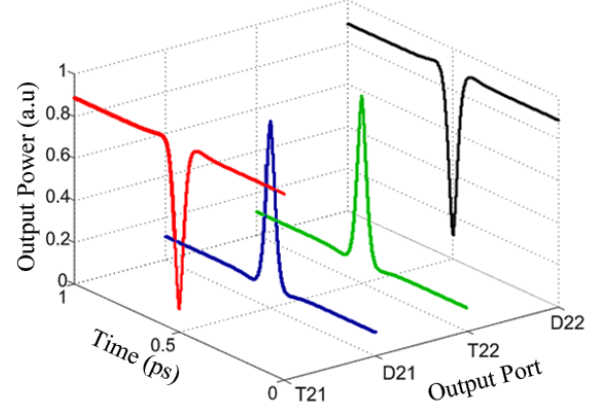

Figure 7. Show the output logic gate when the input logic stages ' 11 ' or 'BB'.

\section{All Optical S-R Flip-Flop}

S-R flip-flop is a circuit with two cross-coupled NOR gates or two cross-coupled NAND gate. It has two input signals, which one is $\mathrm{S}$ and the other is $\mathrm{R}$ for setting and resetting the outputs $Q_{n+1}$ and $\bar{Q}_{n+1}$, respectively. The S-R flip-flop outputs are the feedback signals. There are three status logic output signals, which is called "Hold" when no input signal $(\mathrm{S}=0, \mathrm{R}=0$ ), "Set" when $\mathrm{S}=1$ and $\mathrm{R}=0$ and "Reset" when $S=0, R=1$, respectively. The proposed scheme for all-optical S-R flip-flop using beam splitter (BS) and beam combiner (BC) is as shown in Figure 8. The input logic ' 1 ' is formed by bright soliton and logic ' 0 ' is formed by absence of the input signal. The other technique that can be used to form the similar operation is found in reference ${ }^{(12)}$, in which the optical power signal is manipulated by erbium doped fiber amplifier (EDFA).

In simulation, the add/drop optical filter parameters are used and fixed to be $K_{s}=0.5, R a d=1.55 \mu \mathrm{m}, A_{\text {eff }}=0.25 \mu \mathrm{m}^{2}$, $\alpha=0.05 \mathrm{dBmm}^{-1}, \gamma=0.01, n_{\text {eff }}=3.14$ (for InGaAsP $/ \operatorname{InP}$ ) for all add/drop optical filters in the system. Results of the all-optical flip-flop are generated by the dark-bright solitons conversion behaviors, with center wavelength is at $\lambda_{0}=1.50 \mu \mathrm{m}$, pulse width of $35 \mathrm{fs}$ and the input data logic " 0 " and " 1 " are represented by the dark and bright soliton pulses, respectively. In Figure 9, the simultaneous output optical logic is seen, which can be configured as following details.

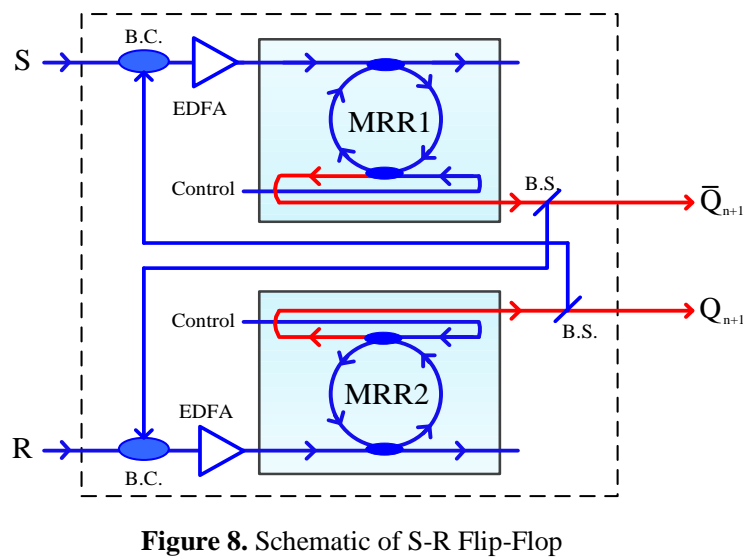

Case 1: When it is no input signal, $S=R=0$, where

$Q_{n+1}$ and $\bar{Q}_{n+1}$ are not changed. This state is called "Hold".

Case 2: By using the dark-bright solitons conversion behavior, when $S=0$ and $R=1$, the input logic ' 0 ' and ' 1 ' is formed by dark-bright solitons, where both input signals are 
converted to dark-bright soliton pulses with $\pi$ phase shift. The detector D2 receives the logic ' 0 ' (dark soliton), i.e.

$Q_{n+1}=0$, which is connected to the input ' $\mathrm{S}$ ', then the dark-bright solitons conversion with $\pi$ phase shift is operated again. When the detector D1 receives the logic ' 1 ' (bright soliton), hence $\bar{Q}_{n+1}=1$, in which $Q_{n+1}$ and $\bar{Q}_{n+1}$ are connected to the input ' $S$ ' and ' $R$ ', respectively. Therefore, if both ' $S$ ' and ' $R$ ' are no input signals, i.e. $\mathrm{S}=\mathrm{R}=0$. Then $Q_{n+1}$ and $\bar{Q}_{n+1}$ are the feedback signals, which is called the "Reset".

Case 3: When $S=1$ and $R=0$, the logic ' 0 ' and ' 1 ' is formed by dark-bright solitons, where both input signals are converted to dark-bright solitons with $\pi$ phase shift. When D1 receives the logic ' 0 ' (dark soliton) i.e. $\bar{Q}_{n+1}=0$, which is connected to the input ' $\mathrm{R}$ ', then the dark-bright solitons conversion with $\pi$ phase shift is formed. When the detector D2 receives the logic ' 1 ' (bright soliton), hence $Q_{n+1}=1$, where $Q_{n+1}$ and $\bar{Q}_{n+1}$ are connected to the input ' $\mathrm{S}$ ' and ' $R$ ', respectively. Therefore, if both ' $S$ ' and ' $R$ ' are no input signals, i.e. $\mathrm{S}=\mathrm{R}=0$. Then $Q_{n+1}$ and $\bar{Q}_{n+1}$ are the feedback signals, which is called the "Set". (See Figure 10)

Case 4: When $S=R=1$ then both MRR1 and MRR2 receive both the incoming bright soliton signals, i.e.

$Q_{n+1}=\bar{Q}_{n+1}$, this state is called the "Forbidden". (See Figure 11)

The truth table for output of the one bit binary R-S Flip-Flop is concluded in Table: 1.

Table 1. Conclusion of the Binary S-R Flip-Flop

\begin{tabular}{|c|c|c|c|c|}
\hline \multicolumn{2}{|c|}{ Input } & \multicolumn{2}{|c|}{ Output } & \multirow{2}{*}{ State } \\
\hline$S$ & $\boldsymbol{R}$ & $Q_{n+1}$ & $\bar{Q}_{n+1}$ & \\
\hline 0 & 0 & $Q_{n}$ & $\bar{Q}_{n}$ & Hold \\
\hline 0 & 1 & 0 & 1 & Reset \\
\hline 1 & 0 & 1 & 0 & Set \\
\hline 1 & 1 & 1 & 1 & Forbidden \\
\hline
\end{tabular}

\section{Simultaneous All-Optical Half Adder/Subtractor}

A binary arithmetic is performed similarly to the decimal arithmetic, which is presented by the logic gate operation, which is as shown in Figure 12. (a). For simplicity, the multiple input ports are required to perform the operation,

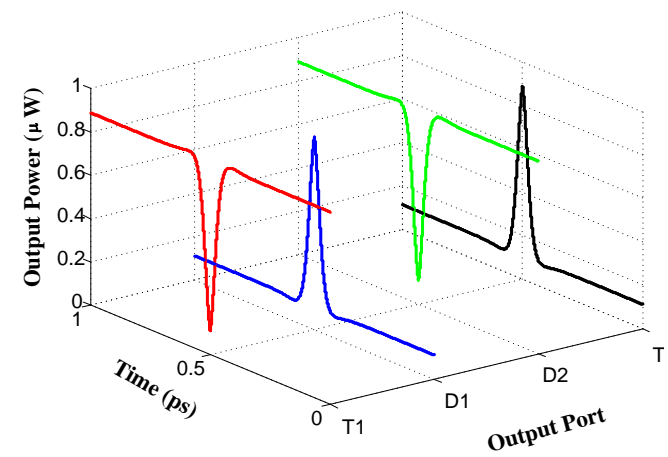

Figure 9. Show the output when 'Reset' stage.

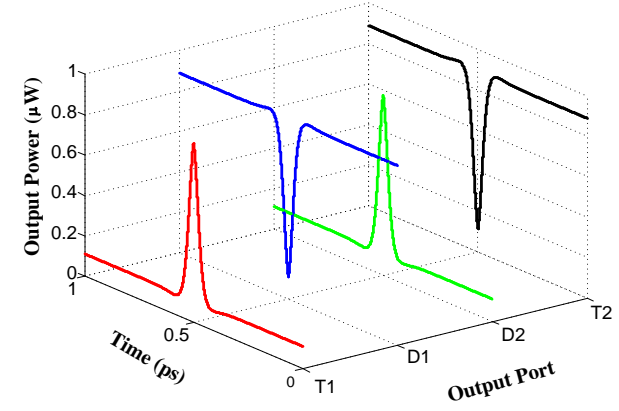

Figure 10. Show the output when 'Set' stage.

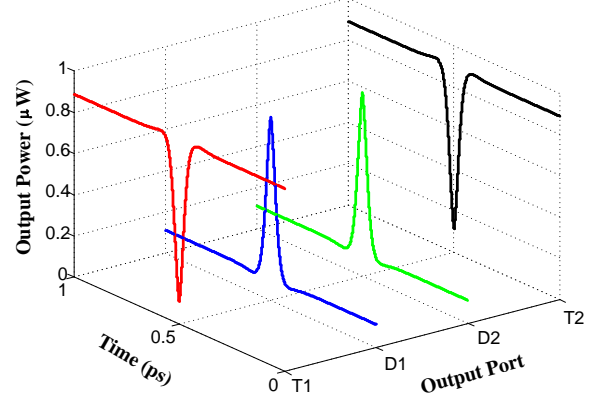

Figure 11. Show the output when 'Set' stage.

where first of all, the required half adder/subtractor truth table is given and shown in Figure 12. (b).For the half adder/subtractor with two binary inputs, the simplified Boolean equation is obtained, in which is the sum of product for half adder and half subtractor, respectively. The simplified output of Sum and Difference can be also implemented with the XOR gate, in which the addition and subtraction operations can be combined into one circuit with one common binary adder. To generate the all-optical half adder/subtractor, it can be easily done by using a beam splitter (BS) or beam combiner (BC), as shown in Figure 3, the same beam splitter is also used in this case. 

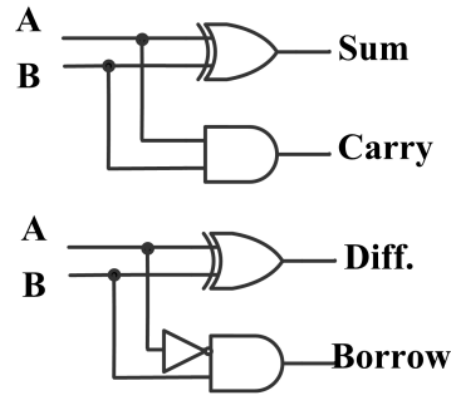

(a)

\begin{tabular}{cc|cc|cc} 
A & B & Sum Carry & \multicolumn{2}{|c}{ Diff. Borrow } \\
\hline 0 & 0 & 0 & 0 & 0 & 0 \\
0 & 1 & 1 & 0 & 1 & 1 \\
1 & 0 & 1 & 0 & 1 & 0 \\
1 & 1 & 0 & 1 & 0 & 0 \\
\hline
\end{tabular}

(b)

Figure 12. (a) schematic of a logic-gate half adder/substractor, (b) a half adder/substractor truth table

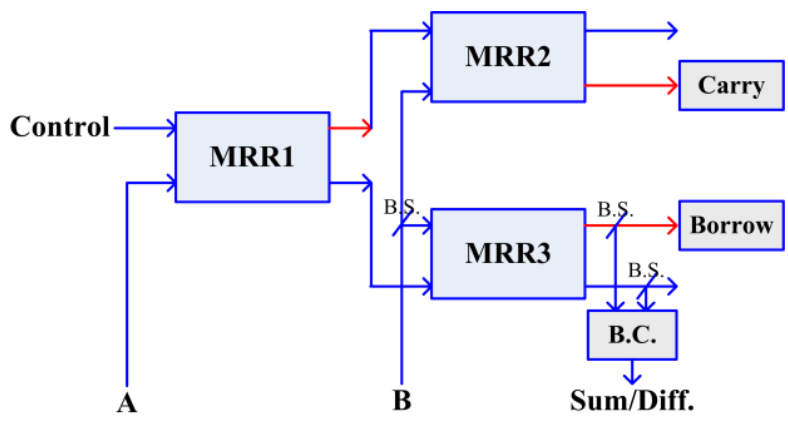

Figure 13. all-optical half adder/substractor

\section{Conclusion}

We proposed photonic circuit can be used to create the logic NAND gate and a new designed for basic all-optical flip-flop and arithmetic operations based on dark-bright solitons conversion control system via the modified add/drop filters for ultrahigh speed memory or signal processing application. Based on the dark-bright solitons conversion control concept, the data logic ' 0 ' (dark soliton) and ' 1 ' (bright soliton) using all-optical in nature can be used to form the flip-flop operations, in which the logic status results can be obtained simultaneously at the drop and through ports, respectively. The all-optical device can perform high capacity optical logic NAND at output port the T21. And the proposed design can be used for logical circuit which is recognized as the simple and flexible system for performing the logic switching system and the device can be extended and implemented for any higher number of input digits by a proper incorporation of dark-bright solitons conversion control.

\section{References}

(1) F. Ramos, E. Kehayas, J. M. Martinez, R. Clavero, J. Marti, L. Stampoulidis, D. Tsiokos,H. Avramopoulos, J. Zhang, P. V. Holm-Niesen, N. Chi, P. Jeppersen, N. Caenegem, D. Colle, M. Pickavet, and B. R. Ti, "IST-LASAGNE: Towards all-optical label swapping employing optical logic gates and optical flip-flops", IEEE Light. Tech., 23, 2993-3011, 2005

(2) M. T. Hill, A. Srivatsa, N. Calabretta, Y. Liu, H. d. Waardt, G. D. Khoe, and H. J. S. Doren, "1x2 optical packet switch using all-optical header processing", Electron. Lett., 37, 774-775, 2001

(3) D. K. Maity, T. Chattopadhyay, D. K. Gayen, C. Taraphdar, A. K. Maiti, S. P. Maity, J. N. Roy, "All-Optical binary Flip-Flop with the help of Terahertz Optical Asymmetric Demultiplexer," Nat. Comput., 9, 903-916, 2010

(4) D. Song, V. Gauss, H Zhang, M. Gross, P. Wen, S. Esener, "All-optical flip-flop based on vertical cavity semiconductor optical amplifiers", Opt. Lett., 32, 29692971, 2007

(5) Y. D. Jeong, J. S. Cho, Y. H. Won , "All-optical flip-flop based on the bistability of injection locked Fabry-Perot laser diode", Opt. Exp., 14, 4058-4063, 2006

(6) E. Tangdiongga, X. Yang, Z. Li, Y. Liu, D. Lenstra, G. D. Khoe, H. J. S. Dorren, "Optical flip-flop based on two-coupled mode-locked ring lasers", IEEE Photon. Technol. Lett., 17, 208-210, 2005

(7) A. Shinya, S. Mitsugi, T. Tanabe, M. Notomi, I. Yokohamma, "All-optical flip-flop circuit composed of coupled two-port resonant tunneling filter in two-dimensional photonic crystal slab". Opt. Express., 14, 1230-1235, 2006

(8) C. H. Chen, S. Matsuo, K. Nozaki, A. Shinya, T. Sato, Y. Kawaguchi, H. Sumikura, M. Notomi, “All-optical memory based on injection-locking bistability in photonic crystal lasers", Optics Exp., 19 (4), 3387-3395, 2011

(9) K. Huybrechts, R. Baets, G. Morthier, "All-optical flip-flop operation in a standard tunable DBR laser diode," IEEE Photon. Technol. Lett., 21(24), 18731875, 2009

(10) S. Mukhopadhyay, J. N. Roy, S. K. Bera, "Design of minimized LED array for maximum parallel logic operations in optical shadow casting technique," Opt. Commun., 99, 31-37, 1993 
(11)E. Rumelhart, J. L. McClelland, Eds., "Parallel Distributed Processing : Explorations in the Microstructure of Cognition," Vols. (1)(2), MIT Press, Boston, 1986

(12) N. Peyghambarian, H. M. Gibbs, "Optical bistability for optical signal processing and computing," Opt. Eng. 24(1), 68-72, 1985

(13) N. Pahari, D. N. Das, S. Mukhopadhyay, "All-optical method for the addition of binary data by nonlinear materias," Appl. Opt., 43(33), 6147-6150, 2004

(14) K. Sarapat, N. Sangwara, K. Srinuanjan, P. P. Yupapin, "Novel dark-bright optical solitons conversion system and power amplification," Opt.Eng. 48(4), 045004-7, 2009

(15) S. Mitatha, "Dark soliton behaviors within the nonlinear micro and nanoring resonators and applications," Progress In Electromagnetics Research, 99, 383-404, 2009

(16) S. Mitatha, N. Chaiyasoonthorn, P. P. Yupapin, "Dark-bright optical solitons conversion via an optical add/drop filter," Microw. and Opt. Technol. Lett., 51, 2104-2107, 2009 